\title{
LOWER BOUNDS FOR THE GROUND STATE ENERGY OF SPIN SYSTEMS
}

\author{
P.M. van den BROEK \\ Department of Applied Physics, Twente University of Technology, Enschede, The Netherlands
}

Received 24 September 1980

\begin{abstract}
A new method is presented for the calculation of lower bounds for the ground state energy of spin systems, using the translational symmetry of the hamiltonian. Numerical results are given for the antiferromagnetic square lattice and the antiferromagnetic triangular lattice, both with Heisenberg nearest neighbour interaction.
\end{abstract}

In this note we will present a new method for the calculation of lower bounds for the ground state energy of spin systems. For convenience we will derive the method for the antiferromagnetic square lattice with Heisenberg nearest neighbour interaction, the generalisation to other spin systems being obvious. The hamiltonian is

$H=\sum_{(i, j)} \boldsymbol{s}_{i} \cdot \boldsymbol{s}_{j}$,

where $i$ and $j$ denote the sites of the square lattice and the summation is over nearest neighbours only. The energy per spin in the ground state has been approximated in several ways by several authors; see ref. [1] for a list of references.

The square lattice has a two-dimensional translational symmetry; let $G$ be the two-dimensional translation group. Then any element $T$ of $G$ commutes with $H$ and

$$
T S_{i} \cdot S_{j} T^{-1}=S_{i^{\prime}} \cdot S_{j^{\prime}},
$$

if $T$ translates the sites $i$ and $j$ to $i^{\prime}$ and $j^{\prime}$ respectively. The ground states of $H$ transform under translations as the basis vectors of a representation of $G$. Since all irreducible representations of $G$ are one-dimensional we can select a ground state $|0\rangle$ which transforms as a basis vector for a one-dimensional representation of $G$. Then

$\left\langle 0\left|S_{i} \cdot S_{j}\right| 0\right\rangle=\left\langle 0\left|S_{i} \cdot S_{j} \cdot\right| 0\right\rangle$,

since $T|0\rangle$ is equal to $|0\rangle$ up to a phase factor.

So all bonds which are related by a symmetry trans- lation have the same expectation value in the state $|0\rangle$. Here all "horizontal" bonds are related by a symmetry translation and also all "vertical" bonds. Let $E_{1}$ and $E_{2}$ be the expectation values of "horizontal" and "vertical" bonds respectively in the state $|0\rangle$, and let $E$ be the ground state energy per spin. Then

$E=E_{1}+E_{2}$.

Now consider a cluster of a finite number of spins and the operator

$K=\sum_{(i, j)} A(i, j) S_{i} \cdot S_{j}+\sum_{(i, j)} B(i, j) \boldsymbol{S}_{i} \cdot \boldsymbol{S}_{j}$,

where $i$ and $j$ are lattice sites in the cluster, $A(i, j)$ and $B(i, j)$ are real numbers subject to the conditions

$\sum_{(i, j)} A(i, j)=\sum_{(i, j)} B(i, j)$

and

$\sum_{(i, j)} A(i, j)>0$

and the first summation contains only "horizontal" bonds in the cluster and the second summation only "vertical" bonds. Then we have

$\langle 0|K| 0\rangle=E \sum_{(i, j)} A(i, j)$.

Let $E(K)$ be the lowest eigenvalue of $K$, then we have $E(K) \leqslant\langle 0|K| 0\rangle$ 
and therefore

$E \geqslant E(K) / \sum_{(i, j)} A(i, j)$.

So the right-hand side of this expression is a lower bound for $E$. The crucial point of this method is the freedom one has for the choice of $A(i, j)$ and $B(i, j)$. These numbers can be chosen in such a way that the best lower bound is obtained. Of course the cluster of spins should be chosen so small that $E(K)$ can be found exactly.

Consider for instance the following cluster of 8 spins:

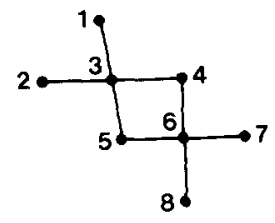

Let $K$ be given by

$$
\begin{aligned}
& K(\gamma)=S_{3} \cdot S_{4}+S_{3} \cdot S_{5}+S_{4} \cdot S_{6}+S_{5} \cdot S_{6} \\
& +\gamma\left(S_{1} \cdot S_{3}+S_{2} \cdot S_{3}+S_{6} \cdot S_{7}+S_{6} \cdot S_{8}\right) .
\end{aligned}
$$

Here the numbers $A(i, j)$ and $B(i, j)$ have been chosen to be the same for "similar" bonds in the cluster, leaving one variation parameter $\gamma$. The total number of horizontal and vertical bonds in the cluster is equal to $2+2 \gamma$. So for each $\gamma>-1$ a lower bound $E_{L B}(\gamma)$ is given by

$E_{\mathrm{LB}}(\gamma)=E(K(\gamma)) /(2+2 \gamma)$.

Numerical calculation shows that the best lower bound is obtained for $\gamma=0.884$ and is equal to -2.952 .

As a second example consider the antiferromagnetic triangular lattice with Heisenberg nearest neighbour interaction. Let $|0\rangle$ be a ground state where all bonds related by a symmetry translation have the same expectation value, and let $E_{1}, E_{2}$ and $E_{3}$ be the expectation values for the three different types of bonds. Then
$E=E_{1}+E_{2}+E_{3}$.

Consider the following cluster

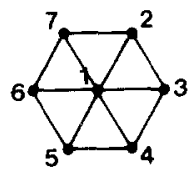

This cluster has been used in ref. [2] for the calculation of an upper bound $(-1.820)$ for $E$. Let $K$ be given by

$K(\gamma)=\sum_{i=2}^{7} S_{1} \cdot S_{i}+\gamma \sum_{i=2}^{6} S_{i} \cdot S_{i+1}+\gamma S_{2} \cdot S_{7}$

This operator contains $2+2 \gamma$ bonds of each type; for each $\gamma>-1$ a lower bound $E_{\mathrm{LB}}(\gamma)$ is given by

$E_{\mathrm{LB}}(\gamma)=E(K(\gamma)) /(2+2 \gamma)$,

where $E(K(\gamma))$ denotes again the lowest eigenvalue of $K(\gamma)$. Numerical calculation shows that the best lower bound is obtained for $\gamma=0.309$ and is equal to -2.528

The two previous methods for calculating lower bounds can also be applied with this cluster. With the method described in ref. [3] one obtains the lower bound $E_{\mathrm{LB}}(1)$ which is equal to -3.118 . The method of overlapping clusters $[4,5]$ gives the lower bound $E_{\mathrm{LB}}(1 / 2)$ which equal to -2.745 . This shows that our method is able to produce, with the same cluster of spins, better lower bounds than the previous methods.

\section{References}

[1] P.M. van den Broek, W.J. Caspers and M.W.M. Willemse, Physica 104A (1980) 298.

[2] H.P. van de Braak, W.J. Caspers and M.W.M. Willemse, Phys. Lett. 67A (1978) 147.

[3] H.P. van de Braak, W.J. Caspers, C. de Lange and M.W.M. Willemse, Physica 87A (1977) 354.

[4] H.P. Bader and R. Schilling, Phys. Rev. B19 (1979) 3556.

[5] P.M. van den Broek, Phys. Lett. 77A (1980) 261. 